\title{
Dentin bonding and physicochemical properties of adhesives incorporated with epigallocatechin-3-gallate
}

\author{
Francisco Adeilson Alves de Macedo ${ }^{1}$ - Nayara O. Souza ${ }^{1}$ Marcelo Victor Sidou Lemos ${ }^{1}$. Diego Martins De-Paula ${ }^{1,2}$. \\ Sergio Lima Santiago ${ }^{1} \cdot$ Victor Pinheiro Feitosa ${ }^{1,2}$ (I)
}

Received: 19 January 2018 / Accepted: 1 May 2018

(C) The Society of The Nippon Dental University 2018

\begin{abstract}
The aim was to assess dentin adhesion and physicochemical properties of experimental etch-and-rinse and self-etch adhesives doped with epigallocatechin-3-gallate (EGCG), a well-known collagen crosslinker obtained from green tea (Camellia sinensis). Experimental adhesives were prepared without (0 wt \%), with 0.1 or $0.5 \mathrm{wt} \%$ EGCG addition. Their degree of conversion was surveyed by FTIR, and bar-shaped specimens were tested to obtain flexural strength and modulus initially and after 1-week ethanol storage. Extracted human molars were prepared, bonded, and cut into resin-dentin sticks for microtensile bond strength test, which was conducted after $24 \mathrm{~h}$ or 6 -month water storage. Statistical analyses were performed with two-way ANOVA and Tukey's test $(p<0.05)$. Degree of conversion outcomes depicted a significant polymerization reduction by the addition of EGCG in self-etch adhesive in both concentrations. However, only $0.1 \%$ reduced the conversion of etch-and-rinse adhesive. Flexural modulus and strength were significantly diminished $(p<0.05)$ by the addition of both concentrations of EGCG for the two model adhesives. Dentin-bond strength was reduced after aging with the addition of EGCG to self-etch adhesive. Nevertheless, $0.5 \%$ EGCG increased the bond strength of etch-and-rinse adhesive after aging, conversely to the significant reduction for EGCG-free control adhesive. In conclusion, EGCG at $0.5 \%$ provides optimal improvements on dentin bonding without altering final polymerization of a model etch-and-rinse adhesive.
\end{abstract}

Keywords Bond strength $\cdot$ Dental adhesive $\cdot$ Green tea $\cdot$ Elastic modulus $\cdot$ Dentin

Victor Pinheiro Feitosa

victorpfeitosa@hotmail.com; victor.feitosa@facpp.edu.br

Francisco Adeilson Alves de Macedo

adeilson.odonto@gmail.com

Nayara O. Souza

nayaraoliv.d@gmail.com

Marcelo Victor Sidou Lemos

marcelosidou@hotmail.com

Diego Martins De-Paula

dmartins1987@hotmail.com

Sergio Lima Santiago

sergiosantiago@yahoo.com

1 Department of Restorative Dentistry, Dental School, Federal University of Ceara, Rua Monsenhor Furtado S/N, Bairro

Rodolfo Teófilo, Fortaleza, CE 60430-325, Brazil

2 Paulo Picanço School of Dentistry, Fortaleza, Brazil

\section{Introduction}

Most contemporary restorative techniques in Dentistry rely on the bonding of resin-based materials to tooth structure. Such bonding is intended to form micromechanical interlocking of polymer chains into the etched enamel and dentin [1-3]. In enamel, the conditioning agent removes a micrometric layer of mineral, thereby creating microretentions for the infiltration of adhesive resin [3, 4]. Conversely, the heterogeneity nature of dentin is associated with impaired resin monomers diffusion and inefficient bond stability, particularly due to the presence of water [5] which promotes hydrolytic degradation of most interface components that might be accelerated by different enzymes [6].

Concerning the bonding mechanism to dentin, micromechanical retention in intertubular dentin, chemical interaction of acidic monomers, and an effective hybridization are considered the most important structures to provide stable dentin bonds [7]. This occurs when the collagen exposed by acid etching is encapsulated by monomers forming a 
homogeneous entanglement of collagen fibrils and polymers chains, the so-called hybrid layer $[8,9]$.

The areas of resin-sparse collagen are considered the threshold for the process of resin-dentin-bond degradation, which might be accelerated by the activity of endopeptidases such as matrix metalloproteinases (MMPs) and cysteine cathepsins $[1,3,10]$. The use of MMP inhibitors has shown to be an useful approach to increase the stability of the hybrid layer, thereby enhancing the durability of the bonding procedures [11-13].

A known plant-derived MMP inhibitor is the epigallocatechin-3-gallate (EGCG) which is the main polyphenol found in green tea (Camellia sinensis) [14]. EGCG has great scientific interest in dentin bonding due to its potential antimicrobial activity against Gram-positive and Gram-negative bacteria [15] and mainly due to its ability to inhibit MMP-2 and MMP-9 [16]. The use of 0.1\% EGCG as a dentin pretreatment solution associated with a two-step etch-and-rinse adhesive resulted in the maintenance of dentin-bond strength after 6 months water storage [17]. Although such reports are relevant, the application of an additional step during adhesive application is clinically unsuitable. The incorporation of EGCG in the adhesives solution may, thus, be an important advantage to avoid also increase on technique sensitivity and application time of adhesive procedure and to produce an adhesive with MMP inhibition properties, thereby protecting the resin-sparse collagen. Some investigations conducted recently showed that EGCG is beneficial in maintaining the integrity of the hybrid layer $[17,18]$. However, there is still limited information about the effects of EGCG addition to model self-etch and etch-and-rinse adhesives on the physicochemical properties and dentin bonding durability.

Therefore, the objective of this in vitro study was to evaluate the adhesion and physicochemical properties of model simplified dental adhesives doped with different concentrations ( 0.1 and $0.5 \mathrm{wt} \%)$ of EGCG. The study hypothesis is that the addition of EGCG in both the experimental self-etch and etch-and-rinse adhesives does not interfere on the degree of conversion, flexural strength, modulus of elasticity, and microtensile dentin-bond strength.

\section{Materials and methods}

\section{Formulation of experimental adhesives}

Two model adhesives were prepared without addition of EGCG. An etch-and-rinse adhesive resin was prepared by mixing $30 \mathrm{wt} \%$ bisphenol-A-glycidyl-dimethacrylate (BisGMA), $17.5 \mathrm{wt} \%$ urethane dimethacrylate (UDMA), $20 \mathrm{wt} \%$ ethanol, $10 \mathrm{wt} \%$ triethylene glycol dimethacrylate (TEGDMA), and $20 \mathrm{wt} \%$ hydroxyethyl methacrylate (HEMA). Model self-etch adhesive was prepared with
$10 \mathrm{wt} \%$ BisGMA, $17.5 \mathrm{wt} \%$ UDMA, $10 \mathrm{wt} \%$ TEGDMA, $20 \mathrm{wt} \%$ ethanol, $10 \mathrm{wt} \%$ HEMA, $15 \mathrm{wt} \%$ distilled water, and $15 \mathrm{wt} \%$ of glycidyl-dimethacrylate phosphate (GDMA-P). In both experimental resins, after mixing co-monomer blend, ternary photoinitiator system was dissolved consisting of $0.5 \mathrm{wt} \%$ camphorquinone (CQ, photosensitive agent), $1 \mathrm{wt} \%$ ethyl-dimethylamine-benzoate (EDAB, tertiary amine), and $1 \mathrm{wt} \%$ diphenyl-iodonium-hexafluorophosphate (DPIHP, accelerator). These two control adhesives were mixed with 0.1 or $0.5 \mathrm{wt} \%$ EGCG (Sigma Aldrich, St. Louis, USA) to obtain experimental therapeutic adhesives.

\section{Degree of conversion}

The degree of conversion of the adhesive resins was evaluated by means of Fourier-transform infrared spectrophotometer (FTIR Spectrometer Bruker, Bruker Co., Bremen, Germany) equipped with attenuated total reflectance (ATR) device with an angle of mirror 45 (PIKE Technologies, WI, USA). A support was coupled to fix the light-curing unit near the spectrophotometer thereby enabling standardization in $3 \mathrm{~mm}$ distance between end of the optical fiber tip and the specimen. Each specimen was dispensed directly onto the ATR crystal in small drops and photoactivated for $40 \mathrm{~s}$ with DB 685 (Dabi Atlante, São Paulo, Brazil) curing unit with $1100-\mathrm{mW} / \mathrm{cm}^{2}$ irradiance.

Three evaluations were conducted for each experimental adhesive and control. The degree of conversion was determined by the ratio of double link $1635 / 1608 \mathrm{~cm}^{-1}$ peaks from aliphatic vinyl methacrylate double bond and aromatic double bond $\left(1608 \mathrm{~cm}^{-1}\right)$ which was used as an internal reference. The values were submitted to two-way ANOVA (type of adhesive and amount of EGCG) and Tukey's test $(p<0.05)$ after passing normality test $(p=0.381)$.

\section{Flexural strength and modulus}

Bar-shaped specimens (1-mm width $\mathrm{x} 1$-mm thickness $\times 7$-mm length) were prepared by inserting the adhesive solutions in silicone molds, following the ISO 4049 protocol [19], except for size of specimen. Light-curing was conducted for $40 \mathrm{~s}$ with DB 685 (Dabi Atlante). The specimens were carefully removed from the molds and evaluated in stereomicroscope, those with defects were discarded. For each group, 12 specimens were prepared $(n=12)$, which half were stored for $24 \mathrm{~h}$ in $100 \%$ relative humidity at $37{ }^{\circ} \mathrm{C}$ or the other half were stored in $90 \%$ ethanol for 1 week at room temperature $\left(25^{\circ} \mathrm{C}\right)$. After storage, flexural strength and elastic modulus were obtained by three-point bending test with $0.5-\mathrm{mm} / \mathrm{min}$ crosshead speed and $5-\mathrm{mm}$ distance between supports, in a Universal Testing Machine (Instron Model 3345, Instron Co., Canton, USA). The bending test was monitored by the Bluehill 2 software (Instron 
Corporation, Canton, USA) which generated a stress-strain curve graph and transformed the data in MPa. The values were statistically analyzed by two-way ANOVA (adhesive type and EGCG concentration) and Tukey's test at 5\% significance level, after proving normal data $(p=0.261$ and $p=0.222$ for elastic modulus and flexural strength, respectively).

\section{Microtensile bond strength}

Thirty extracted human third molars were used after approval by the institutional Research Ethics Committee. They were stored in $0.01 \%$ thymol solution at $4{ }^{\circ} \mathrm{C}$ for no longer than 2 months. Teeth were sectioned $2 \mathrm{~mm}$ above and $2 \mathrm{~mm}$ below cementoenamel junction, to remove the occlusal enamel and roots, and to expose flat middle dentin. They were cut using a diamond blade in a cutting machine (Isomet 1000, Buehler, Lake Bluff, USA). Exposed dentin was polished with 600-grit SiC paper for $30 \mathrm{~s}$ under water irrigation to obtain standardized smear layer.

Thereafter, specimens were randomly divided into 6 groups $(n=5)$. Adhesive application was similar regardless the presence of EGCG. Self-etch adhesives were actively applied for $20 \mathrm{~s}$ on dentin surface, followed by slight air-drying for $5 \mathrm{~s}$ and then photopolymerized for $40 \mathrm{~s}$. For etch-andrinse adhesives, $37 \%$ phosphoric acid etching was realized for $15 \mathrm{~s}$ and washed with distilled water for $30 \mathrm{~s}$, and dentin was kept slightly moist with absorbent paper. Adhesive solution was actively applied for $20 \mathrm{~s}$, slightly air-blasted for $5 \mathrm{~s}$ and then photopolymerized for $40 \mathrm{~s}$. Composite build-ups were constructed using Filtek Supreme XT (3M ESPE) in 5 increments of approximately $1.0 \mathrm{~mm}$.

Bonded teeth were cut longitudinally in a cutting machine (Isomet) at low speed under water refrigeration 1-mm-thick slabs, rotated in $90^{\circ}$ and resin-dentin sticks were obtained with approximately $1-\mathrm{mm}^{2}$ area. At this point, half of the sticks from each tooth were immersed in distilled water for $24 \mathrm{~h}$ and tested for microtensile bond strength. Further half were aged for 6 months in distilled water at $37{ }^{\circ} \mathrm{C}$ with monthly water exchange before microtensile test. Pre-test failures were uncommon (less than three per group) and were set and included in statistics as $0 \mathrm{MPa}$.

In microtensile bond strength test, the sticks were glued with cyanoacrylate gel adhesive in microtensile jig and adapted in a universal testing machine (Instron 3345) and tested to tension until fracture with $0.5-\mathrm{mm} / \mathrm{min}$ crosshead speed and $500 \mathrm{~N}$ load cell. Previously to the test, the area of the bonding interface of the sticks was measured with digital calipers (Absolute Digimatic, Mitutoyo, Tokyo, Japan) and data were transformed to megapascals by dividing maximum tensile strength in Newtons by the cross-sectional area. The values were submitted to two-way ANOVA (adhesive and aging period) and Tukey's test $(p<0.05)$ after passing normality test $(p=0.461)$. After microtensile bond strength test, fractured specimens were surveyed in stereomicroscopy with $80 \mathrm{X}$ magnification (Olympus, Tokyo, Japan). The fractures were classified as adhesive, mixed, cohesive in composite, or cohesive in dentin [5].

\section{Results}

The highest degree of conversion was observed with control self-etch adhesive (average $76.5 \%$ conversion) which was statistically higher than all the further adhesive resins $(p<0.05)$. The incorporation of EGCG had an adverse effect on polymerization (Fig. 1) for self-etch adhesive at both concentrations with statistical reduction with $0.1 \%$ EGCG $(p=0.013)$ and for $0.5 \%$ EGCG $(p<0.001)$. For etch-andrinse adhesives, control resin (mean $64.9 \%$ conversion) demonstrated a significant reduction $(p=0.003)$ of conversion only with addition of $0.1 \%$ EGCG (mean $51.6 \%$ conversion), whereas $0.5 \%$ EGCG demonstrated statistical similarity with control and $0.1 \%$ EGCG resins.

The outcomes of elastic modulus are presented in Fig. 2. Statistical reduction on elastic modulus was observed only for etch-and-rinse adhesive with $0.5 \%$ EGCG (mean $144.8 \mathrm{MPa}$ ) in comparison with control EGCG-free etchand-rinse adhesive (mean 303.6 MPa). At 24-h period, the elastic modulus of self-etch adhesives was not influenced by EGCG addition $(p>0.05)$. After 1 week storage in absolute ethanol, the three self-etch adhesives (SEAs) showed no statistical change on modulus ( $p>0.05)$, whilst all etch-andrinse adhesives (ERAs) demonstrated a significant increase $(p<0.05)$. However, ethanol storage induced significant

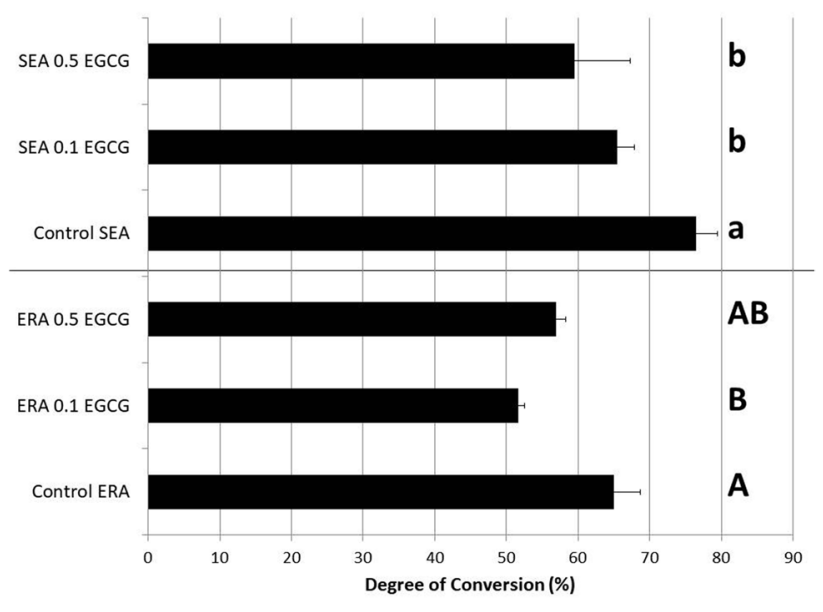

Fig. 1 Degree of conversion results (\%). Different capital letters indicate a significant difference between etch-and-rinse adhesives (ERAs), whilst different tiny letters represent statistical difference between self-etch adhesives (SEAs). SEA self-etch adhesive, ERA etch-and-rinse adhesive, $E G C G$ epigallocatechin-3-gallate 
differences among both adhesive resins. Both concentrations of EGCG attained lower modulus than control SEA $(p<0.05)$, whereas, in ERA, only $0.1 \%$ EGCG had lower modulus than control.

Results of maximum flexural strength (FS) are presented in Fig. 3. The initial FS of control SEA was significantly diminished for both concentrations of EGCG $(p<0.05)$, but for ERA only $0.5 \%$ EGCG adhesive achieved statistical lower FS than control. After ethanol immersion, SEA $+0.5 \%$ EGCG and all three etch-and-rinse adhesives attained increase $(p<0.001)$ on flexural strength. Regarding the intra-group comparison after ethanol storage, no significant difference was found for etch-and-rinse adhesives, and SEA containing $0.1 \%$ EGCG obtained statistically lower FS $(p<0.05)$ than control SEA and SEA with $0.5 \%$ EGCG.

Results from microtensile bond strength assessment are depicted in Table 1. At 24-h analysis, no difference was observed between three SEAs. However, both concentrations of EGCG diminished statistically $(p<0.05)$ the bond strength of etch-and-rinse adhesive. After aging, there was reduction of bond strength for all adhesives, except control self-etch and ERA with 0.1\% EGCG, which demonstrated stable adhesion $(p>0.05)$, and etch-and-rinse adhesive containing $0.5 \%$ EGCG, which attained increase $(p<0.05)$ of bond strength after 6 months water storage. More than 50\% of failure modes were adhesive for all groups, and the percentage of adhesive fractures increased after aging.

\section{Discussion}

The therapeutic effect of EGCG, the main polyphenol found in green tea (Camellia sinensis), has been widely investigated in restorative dentistry, being indicated as a potential
Fig. 2 Results of elastic modulus (MPa). Different uppercase letters show statistical difference $(p<0.05)$ among groups after ethanol immersion. Lowercase letters indicates A significant difference $(p<0.05)$ among groups at 24-h period. $S E A$ self-etch adhesive, $E R A$ etch-and-rinse adhesive, $E G C G$ epigallocatechin-3-gallate
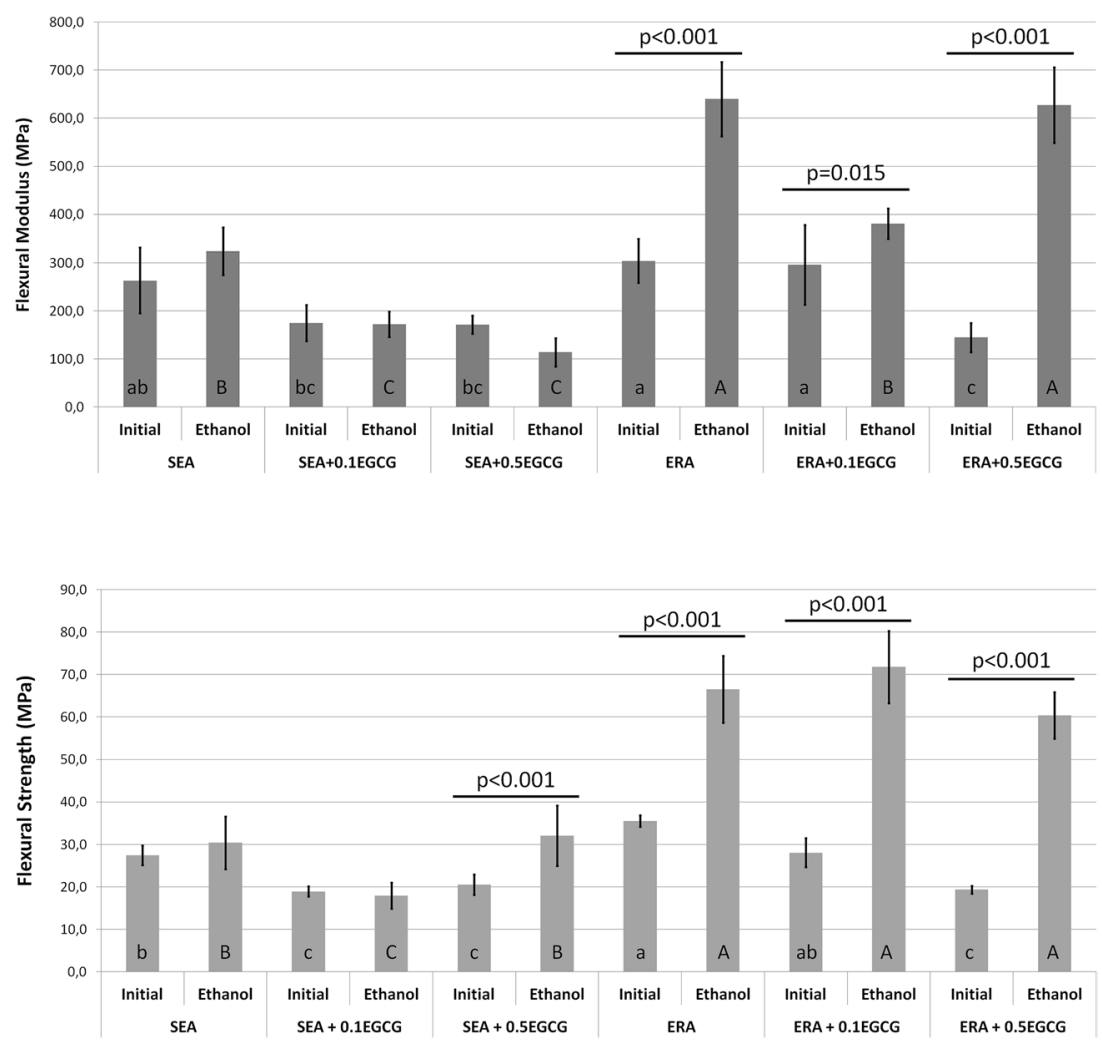

Fig. 3 Results of maximum flexural strength (MPa). Different uppercase letters show statistical difference $(p<0.05)$ among groups after ethanol immersion. Lowercase letters indicates a significant difference $(p<0.05)$ among groups at 24-h period. SEA- self-etch adhesive; ERA- etch-and-rinse adhesive; EGCG: epigallocatechin3-gallate

Table 1 Results of microtensile bond strength $(\mathrm{MPa})$ presented in means (standard deviations)

\begin{tabular}{lllllll}
\hline MPa & SEA & SEA 0.1EGCG & SEA 0.5EGCG & ERA & ERA 0.1EGCG & ERA 0.5EGCG \\
\hline $24 \mathrm{~h}$ & $39.0(9.5) \mathrm{ABa}$ & $47.9(3.4) \mathrm{Aa}$ & $30.7(2.9) \mathrm{Ba}$ & $36.0(7.3) \mathrm{Ba}$ & $25.5(6.1) \mathrm{Ca}$ & $22.0(2.6) \mathrm{Cb}$ \\
$6 \mathrm{~m}$ & $43.9(8.7) \mathrm{Aa}$ & $38.4(3.7) \mathrm{Ab}$ & $17.6(3.9) \mathrm{Bb}$ & $22.3(4.1) \mathrm{Bb}$ & $22.8(4.0) \mathrm{Ba}$ & $44.3(8.0) \mathrm{Aa}$ \\
\hline
\end{tabular}

$6 \mathrm{~m}$ six months, $S E A$ self-etch adhesive, $E R A$ etch-and-rinse adhesive, $E G C G$ epigallocatechin-3-gallate Different capital letters represent a significant difference $(p<0.05)$ among adhesives within the same storage period. Different lower case letters indicate statistical difference $(p<0.05)$ between $24 \mathrm{~h}$ and 6-month outcomes for the same adhesive resin 
inhibitor of matrix metalloproteinases [20] and collagen cross-linking agent [21]. In addition to its antioxidant activity [17], these combined characteristics seem to act to preserve the resin-dentin interface for long periods of aging [17].

According Du et al. [18], EGCG has high antioxidant power with possible negative influence on polymerization and on formation of highly crosslinked polymers. The initial reduction of bond strength noted in microtensile test might be explained by the interaction between camphorquinone/tertiary amine and the flavonoid as reported by Gotti et al. [22] with quercetin. Indeed, this results in decrease of degree of conversion, which may explain the present outcomes (Fig. 1). Nevertheless, no significant diminishing of polymerization was found for etch-and-rinse adhesive doped with $0.5 \%$ EGCG. This fact might be explained by a late polymerization with high antioxidant concentrations where there was a balance between photoinitiator and EGCG (both at $0.5 \%$ concentration). Therefore, subsequent release of EGCG to collagen mesh induces collagen crosslinking [21] and warrants high degree of conversion. The outcomes of flexural strength and modulus of elasticity, which depicted increase after ethanol immersion (Figs. 2, 3), support the occurrence of such late polymerization.

According to Santiago et al. [17], when EGCG is used as pre-treatment in concentrations up to $0.1 \mathrm{wt} \%$, it does not alter the immediate bond strength. Contrariwise, the present investigation depicted immediate bond strength reduction when EGCG was mixed in the blend of model etch-and-rinse adhesive. This might, indeed, be related to the interaction between photoinitiator and antioxidant [22]. The highest solubility of EGCG in water is $0.5 \mathrm{wt} \%$, thereby limiting their incorporation in the adhesive in larger amounts and demonstrating a hydrophobic feature. The study of Gotti et al. [22] found increased bond strength over time with large amounts of antioxidants (5\%) incorporated into different adhesive blend, especially if the antioxidant possesses hydrophobic characteristic. Such increase of bond strength was also observed herein when EGCG was employed in highest concentration $(0.5 \mathrm{wt} \%)$ added to etch-and-rinse adhesive (Table 1). In fact, this outcome demonstrates the extension of resin-dentin-bond longevity and therapeutic effects encountered with the use of EGCG in adhesive dentistry $[13,17]$.

The biomodification attributed to EGCG is due to galloyl functionalities [21], which have high dentin biomodification potential by performing collagen crosslinking. However, the present study was undertaken to incorporate EGCG into adhesives to reduce the clinical application time. In this scenario, it is needed to release EGCG molecules for further action, which explains the increase in bond strength (improvements) only after 6 months aging after application. As for the self-etch adhesive, the thin layer of collagen exposed seems to have minor influence on the longevity of bonds, once both concentrations of EGCG were unable to provide therapeutic effects.

The results obtained in the present investigation are also in disagreement with the findings of Du et al [18], which showed slight decrease in the degree of conversion in adhesive systems containing EGCG 0.01, 0.02 and $0.03 \%$, but without statistical significance, except for the conventional adhesive group containing $0.01 \%$, which showed significant difference. Nevertheless, in that study [18], authors reported difficulties in solubilizing EGCG in higher concentrations. Yet, they related negative interference of EGCG on polymerization when mixed in adhesive blend, thereby interfering in the formation of crosslinked polymer chains and impairing the mechanical properties. Their study is partially in accordance and supports the present findings.

The modulus of elasticity describes the relative stiffness of a material and may be measured by stressXstrain curves. The flexural resistance found in this study was obtained through three-point bending test, and depicted a decrease in the values of the self-etch and etch-and-rinse adhesives incorporated with 0.1 and $0.5 \%$ EGCG compared with the experimental control adhesives (Fig. 3). Concerning elastic modulus, major findings were the drop on the initial stiffness of etch-and-rinse adhesive when adding $0.5 \mathrm{wt} \%$ EGCG (Fig. 2). However, whilst the control (EGCG-free) adhesive had a twofold increase of modulus after ethanol storage, the highest concentration of EGCG afforded a fourfold increase of modulus. Indeed, this may also contribute to the increase of bond strength over time (Table 1) along with the formation of dentin collagen crosslinking [21].

Our outcomes of elastic modulus differed from those of Pallan et al. [13], which noted that the addition of $0.2 \mathrm{wt} \%$ EGCG led to an increase in stiffness, but $1 \mathrm{wt} \%$ showed no statistical improvement on flexural properties. Furthermore, when the concentration of EGCG was increased to $2 \mathrm{wt} \%$, the adhesive resin decreased significantly flexural strength and modulus. The contrasting results may likely be attributed to the use of EGCG in different concentrations and with different purities, which may yield differences in the final amount of EGCG incorporated to the resin blend. Moreover, the difficulty of solubilization of the EGCG in adhesive resins with contrasting hydrophobicity may play a role in the different outcomes obtained.

Once EGCG has modified the dentin bonding, and chemical and mechanical properties of the experimental adhesive resins, the study hypothesis must be rejected. However, the values of physicochemical properties obtained with the addition of EGCG were within the acceptable range when compared with other commercial adhesives. Moreover, EGCG ameliorates the resin-dentin-bond longevity of etch-andrinse adhesive when incorporated in $0.5 \mathrm{wt} \%$, what may 
afford enhancing of adhesive performance without additional clinical steps.

In conclusion, the incorporation of epigallocatechin3 -gallate at 0.1 and $0.5 \%$ in the etch-and-rinse and self-etch experimental adhesives promoted minor changes on physicochemical properties, but with important improvements on flexural strength and modulus as well as on dentin-bond durability when $0.5 \mathrm{wt} \%$ EGCG is added to etch-and-rinse adhesive.

Acknowledgements This investigation was partially supported by grant CNPQ/Universal 2014 Project 457931/2014-0 (PI VPF). We also thank Capes and CNPQ Brazilian agencies for the scholarships provided. We are grateful to Essetech Inc. for the donation of monomers and further reagents.

\section{Compliance with Ethical Standards}

Conflict of interest The authors declare that they have no conflict of interest.

\section{References}

1. Breschi L, Mazzoni A, Dorigo E. Dental adhesion review: aging and stability of the bonded interface. Dent Mater. 2008;24:90-101.

2. Buonocore MG. A simple method of increasing the adhesion of acrylic filling materials to enamel surfaces. J Dent Res. 1955;34:849-53.

3. Nishitani Y, Yoshiyama M, Wadgaonkar B, Breschi L, Mannello F, Mazzoni A. Activation of gelatinolytic/collagenolytic activity in dentin by self-etching adhesives. Eur J Oral Sci. 2006;114:160-66.

4. Loguercio AD, Stanislawczuk R, Polli LG, Costa JA, Michel MD, Reis A. Influence of chlorhexidine digluconate concentration and application time on resin-dentin-bond strength durability. Eur J Oral Sci. 2009;117:587-96.

5. Feitosa VP, Correr AB, Correr-Sobrinho L, Sinhoreti MA. Effect of a new method to simulate pulpal pressure on bond strength and nanoleakage of dental adhesives to dentin. J Adhes Dent. 2012;14:517-24.

6. Pashley DH, Tay FR, Yiu C, Hashimoto M, Breschi L, Carvalho $\mathrm{RM}$, Ito $\mathrm{S}$. Collagen degradation by host-derived enzymes during aging. J Dent Res. 2004;83:216-21.

7. Feitosa VP, Sauro S, Ogliari FA, Ogliari AO, Yoshihara K, Zanchi $\mathrm{CH}$, Correr-Sobrinho L, Sinhoreti MA, Correr AB, Watson TF,
Van Meerbeek B. Impact of hydrophilicity and length of spacer chains on the bonding of functional monomers. Dent Mater. 2014;30:e317-23.

8. Al-Ehaideb A, Mohammed H. Shear bond strength of "one 2 . bottle" dentin adhesives. J Prosthet Dent. 2000;84:408-12.

9. Brackett WW, Ito S, Tay FR, Haisch LD, Pashley DH. Microtensile dentin bond strength of self-etching resins: effect of a hydrophobic layer. Oper Dent. 2005;30:733-8.

10. Garcia RN, Schaible BR, Frankenberger R. Resistência de união de sistemas adesivos autocondicionantes em dentina profunda. RSBO. 2008;3:39-47.

11. Carrilho MRO, Carvalho RM, De Goes MF, DI Hipolito V, Geraldeli S, Tay FR, Pashley DH, Tjaderhane L. Chlorhexidine preserves dentin bond in vitro. J Dent Res. 2007;86:90-4.

12. Maruyama T, Tomofuji T, Endo Y, Irie K, Azuma T, Ekuni D, et al. Supplementation of green tea catechins in dentifrices suppresses gingival oxidative stress and periodontal inflammation. Arch Oral Biol. 2011;56:48-53.

13. Pallan S, Araujo MVF, Prakki A. Mechanical properties and characteristics of developmental copolymers incorporating catechin or chlorhexidine. Dent Mater. 2012;28:687-94.

14. Demeule M, Brossard M, Page M, Gingras D. Matrix metalloproteinase inhibition by green tea catechins. Biochim Biophys Acta. 2000;1478:51-60.

15. Ferrazzano GF, Amato I, Ingenito A, De Natale A, Pollio A. Anticariogenic effects of polyphenols from plant stimulant beverages. Fitoterapia. 2009;80:255-62.

16. Garbisa S, Sartor L, Biggi S, Salvato B, Benelli R, Albini A. Tumor gelatinases and invasion inhibited by the green tea flavanol epigallocatechin-3-gallate. Cancer, 2001;1:822-32.

17. Santiago SL, Osorio R, Neri JR, Carvalho RM, Toledano M Effect of the flavonoid epigallocatechin-3-gallate on resin-dentin bond strength. J Adhes Dent. 2013;15:535-40.

18. Du X, Huang X, Zhang Y. Epigallocatechin-3-gallate (EGCG) enhances the therapeutic activity of a dental adhesive. J Dent. 2012:40:485-92.

19. Standard. ISO 4049. Polymer based filling, restorative and luting materials. Geneva: International Organization for Standardization; 2000. 1-27.

20. Khamverdi Z, Rezaei-Soufi L, Rostamzadeh T. The effect of epigallocatechin gallate on the dentin bond durability of two self-etch adhesives. J Dent (Shiraz). 2015;16:68-74.

21. Vidal CM, Aguiar TR, Phansalkar R, McAlpine JB, Napolitano JG, Chen SN, Araújo LS, Pauli GF, Bedran-Russo A. Galloyl moieties enhance the dentin biomodification potential of plantderived catechins. Acta Biomater. 2014;10:3288-94.

22. Gotti VB, Feitosa VP, Sauro S, Correr-Sobrinho L, Leal FB, Stansbury JW, Correr AB. Effect of antioxidants on the dentin interface bond stability of adhesives exposed to hydrolytic degradation. J Adhes Dent. 2015;17:35-44. 